\title{
Research on a New Large Mowing Robot and Path Planning Based on GPS-RTK
}

\author{
Zhou Jiehua $^{1,2, a}$, Zhou Jiqiang, ${ }^{1, b}$, Zhang Xiaoyong ${ }^{1, c}$ and Kong Bin ${ }^{3, d}$ \\ 1Jiangxi Hongdu Aviation Industry Group Limited Liability Company, Nanchang, China, 330024 \\ ${ }^{2}$ School on Information Engineering, Nanchang Hangkong University, Nanchang, China, 330063 \\ ${ }^{3}$ Institute of Intelligent Machines, Chinese Academy of Sciences, Hefei, China, 230031 \\ ajiehuazhou@126.com, bzjq3921@163.com, ${ }^{\mathrm{c}} 76284491 @ q q . c o m,{ }^{\mathrm{d}}$ bkong@iim.ac.cn
}

Keywords: mowing robot, GPS-RTK, path planning

Abstract. A new mowing robot suitable for large-scale lawn is proposed in the paper. The mowing robot mainly consists of mobile platform, GPS navigation system, control system, power system and remote monitoring system. First of all, the paper introduces the functions of each subsystem from the point of view of system design. Secondly, GPS is used as navigation tool, and GPS-RTK technology is used to realize precise positioning of mowing robot. The tasks of working area build and path planning of robot are completed by GPS information, and taking an airport lawn as the experimental object, the task of working area division was completed.

\section{Introduction}

Under the background of social progress and economic development, people's demand for living environment is also getting higher and higher, and more and more people pay attention to the protection of urban environment. Our lawn construction has obtained rapid development, such as city landscaping, golf course, football field, tennis court, racetrack, rivers, highways, railways and other transportation and water conservancy facilities all need high quality lawn. As you all know, the lawn can beautify the environment, soil consolidation, slope protection, purification of air and so on. In the United States, the lawn industry has become one of its ten pillar industries. According to incomplete statistics, there are 660 thousand hectares green land in 500 cities of China, and the lawn accounted for 180 thousand hectares [1-2]. In all kinds of lawn maintenance work, the lawn pruning work is the most heavy, not only boring, but also repetitive, usually need to consume a lot of manpower and material resources. Therefore, the development of lawn means a large demand for new unmanned mowing vehicles [3-5].

With the rapid development of city, people have higher requirements for the green environment. How to meet the management requirements of a large number of lawns naturally becomes an important research topic. The traditional manual mower has low working efficiency, large noise, high labor intensity and potential safety hazard, so it can't adapt to the development of green construction and a new automated unmanned mowing vehicle to replace the traditional mower is an inevitable trend of the development of green industry. Especially in the large-scale grassland environment, if you just rely on labor for pruning, the labor intensity is very large [2]. Therefore, the application of unmanned mowing vehicles in the large-scale grassland environment will greatly ease the pressure of related organizations on turf management, but also save a lot of time for the lawn managers.

\section{Large mowing robot system}

The large mowing robot studied in this paper is an integrated robot system which integrates environment perception, dynamic path planning and behavior control. It is mainly used in airport, golf course, pasture and so on. Four-wheel drive chassis is used in the mowing robot, and a large cutting table is embedded in the body of robot. The hybrid oil and electric power scheme is adopted, including gasoline engine, generator, AC/DC charging module, battery pack and power management module. The gasoline engine provides all the power sources, and drives the generator to obtain the direct 
current to provide power for the walking motor and other equipment on the vehicle. At the same time, it directly provides mowing power to the cutting table. GPS-RTK navigation equipment is used to obtain accurate position and course information of unmanned mower for path planning and precision navigation. The surrounding environment information of mower is acquired by using radar sensor, which is used to avoid obstacle. The embedded intelligent controller of robot controls the motion of the vehicle, and collects GPS navigation information acquisition, obstacle avoidance sensor information for path planning, navigation, trajectory tracking and so on. The control signal is sent to the corresponding actuator, and the real-time status information is returned to the remote monitoring terminal.

The structure diagram of the autonomous unmanned mowing robot system is shown in Fig. 1, which consists of the following parts:

(1) Mobile platform

The mobile platform includes chassis mechanism, walking executive mechanism, steering executive mechanism and height adjustment mechanism of the cutting table. The walking executive mechanism, steering executive mechanism and height adjustment mechanism of the cutting table all consist of DC servo motors reducer and motor driver. A total of nine sets of motor drivers are connected by CAN bus communication mode for unified control. The mechanical structure of autonomous unmanned mower is shown in Fig. 2. Its chassis is driven by four sets of DC motors driving four wheels, and the front two wheels control the steering of the vehicle. The height of the cutting table can be adjusted from eight centimeters to thirty centimeters.

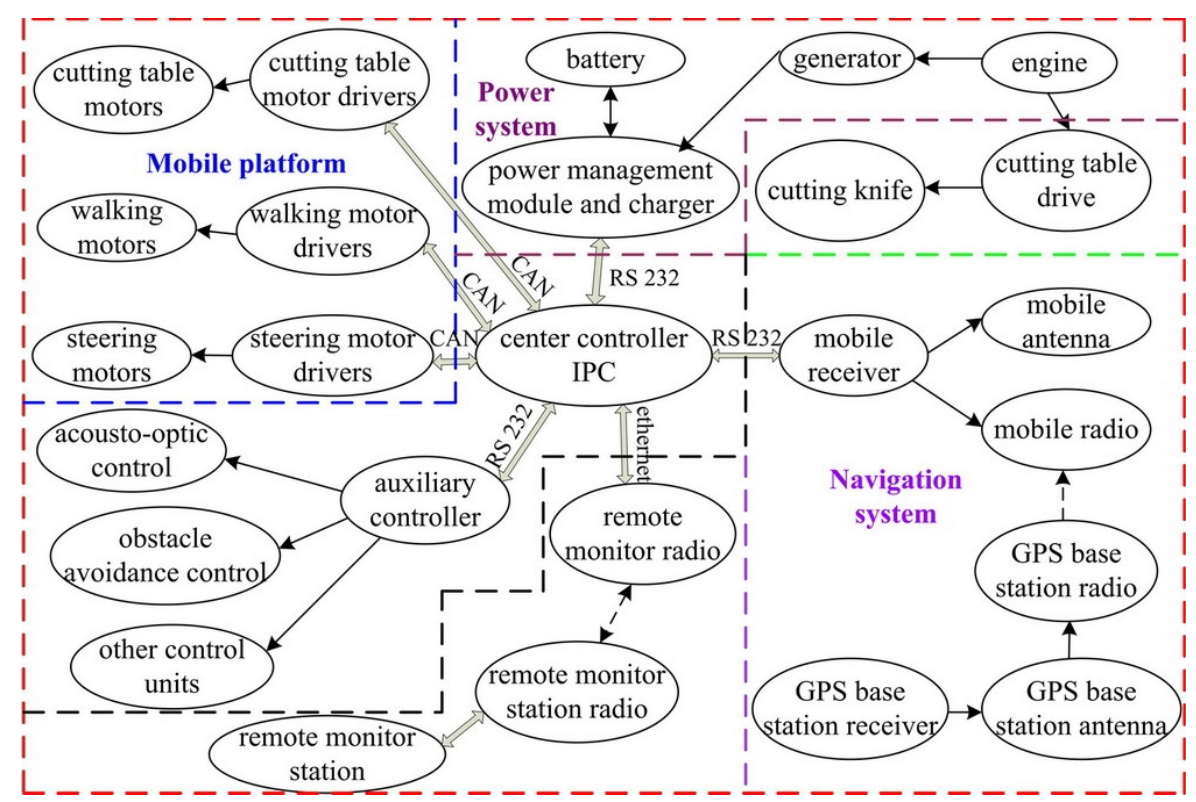

Fig. 1 Structure diagram of the autonomous unmanned mowing robot system

(2) GPS-RTK navigation technology

The robot uses GPS-RTK navigation technology to obtain accurate position and course information of unmanned mower for path planning and precision navigation. Navigation system shown in Fig. 3 consists of GPS mobile station and GPS base station. The base station including satellite antenna, base station receiver and transmitting radio station is placed in the fixed position. The mobile station including the satellite antenna, the mobile station receiver and the receiving radio station is placed on the mower. The GPS-RTK navigation system consisting of GPS-RTK base station and mobile station can achieve pinpoint of centimeter level. The functions of the two stations are as follows:

1) GPS-RTK base station obtains the fixed point position signal through single GPS device, and sends the differential signal to the mobile station to carry on the differential computation. The GPS-RTK base station consists of base receiver, satellite signal receiving antenna, digital radio, digital radio antenna, $12 \mathrm{~V}$ DC power supply equipment and so on. 
2) GPS-RTK mobile station performs differential operation to obtain pinpoint of centimeter level based on the position information obtained by the mobile station and differential position signals transmitted by the base station. The construction of the GPS-RTK mobile station requires GPS-RTK receiver, two satellite antennas, digital radio receiver, digital radio antenna, $12 \mathrm{~V}$ DC power supply and vehicle.

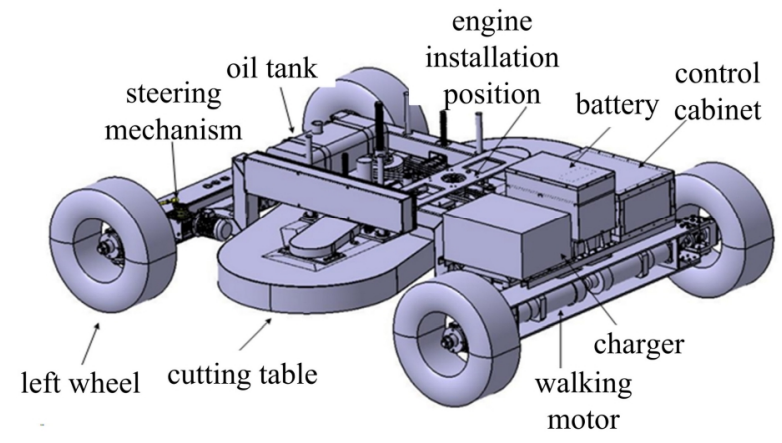

Fig. 2 Mechanical structure of autonomous unmanned mower

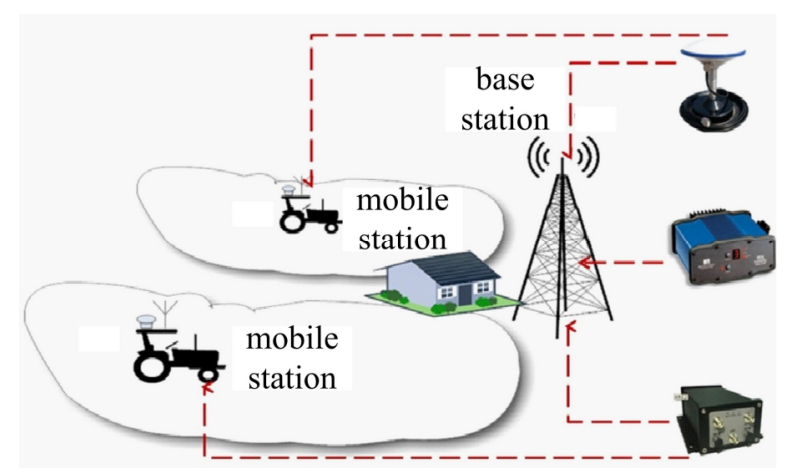

Fig. 3 Navigation system

(3) Control system

The industrial personal computer or mobile intelligent controller is used to build the center controller of robot which is used to output motor drive instructions, collect GPS navigation information, remote monitoring communication information, power management system data and auxiliary control instructions. Auxiliary control module includes sound and light control, obstacle avoidance, remote control interface and engine control.The interface structure of the mower control mainly includes GPS communication interface, remote monitoring communication interface, motor communication interface, handshake communication interface and AI, DI, DO communication interface. Each interface function is as follows:

1) GPS communication interface is mainly used to collect accurate positioning information of GPS output.

2) The remote monitoring communication interface is mainly connected with the remote monitoring communication station, which can send the real-time state to the remote monitoring terminal and receive the control command of the remote monitoring terminal.

3) The motor communication interface is used to send control commands to the motor of the walking, steering and cutting table.

4) The handshake communication interface is used to judge whether the industrial computer crashes or not and executes the corresponding exception handling actions.

5) The AI, DI and DO communication interfaces used to realize the functions of analog acquisition, switch collection and output are built by the special serial port control board module.

(4) Power system

Fig. 4 shows the power system scheme of unmanned mower. The power system of unmanned mower adopts hybrid oil and electric power scheme, which consists of gasoline engine, generator, charger, battery pack and power management module. The gasoline engine provides total power source. It provides the mowing power directly to the mower cutting table through the transmission mechanism, and connects the generator through the belt to output the direct current and charge the battery pack. Finally, the power of mower and the electric equipment on the vehicle are jointly supplied by the generator and the battery pack. The output voltage of the generator is AC $220 \mathrm{~V}$, and the output DC $180 \mathrm{~V}$ of the charger is supplied to the walking motor and other electrical equipment. When the generator fails or fails to work, the power is directly supplied by the battery pack.

(5) Remote monitoring system

The ordinary PC is used to build remote monitoring terminal of unmanned mower. The video surveillance equipment is installed on the unmanned mower to obtain video monitoring information, and the video signal, mower status and other information transmitted to the remote monitoring 
terminal through high performance digital radio. At the same time, we can develop the corresponding application software on the remote monitoring terminal. As shown in Fig. 5, the remote monitoring schematic diagram of unmanned mower is adopted, and the high performance network radio is used as the remote communication medium. The remote monitoring system is mainly composed of two communication links, one of which is the common link, mainly used to transfer state information and control instructions, can realize half duplex communication. The other one is the special real-time video transmission link, can realize one-way communication.

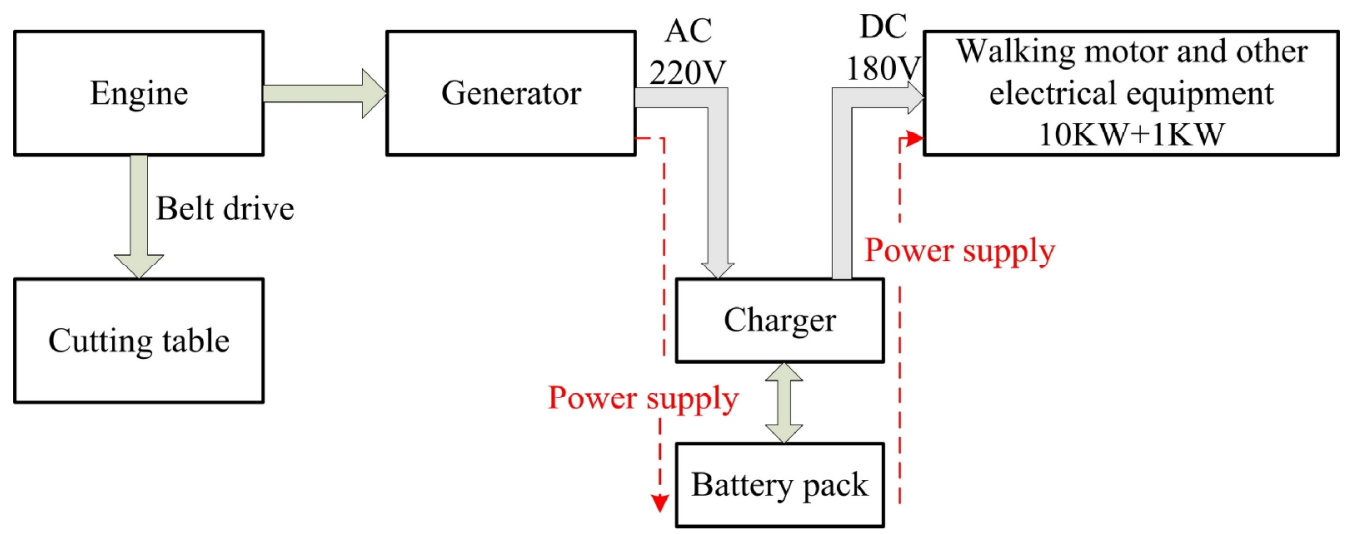

Fig. 4 Power system scheme of unmanned mower

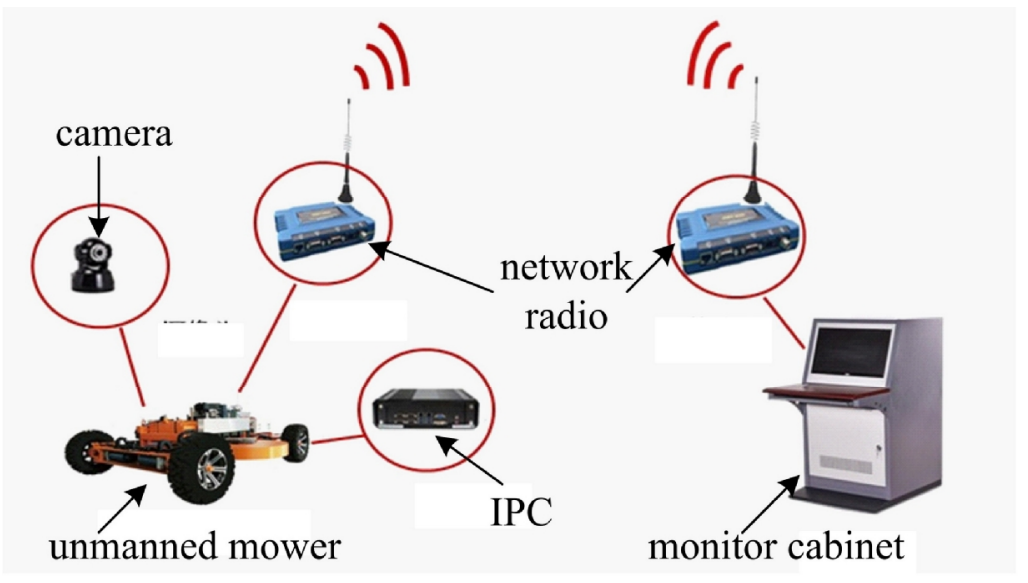

Fig. 5 Remote monitoring schematic diagram of unmanned mower

\section{Path planning}

Path planning algorithm design. Path planning is the key factor to achieve high efficiency working ability for mowing robot. The traditional path planning is to seek a optimal path having no collision from the start point to the end point. The traditional path planning types of mowing robot are random, spiral and roundabout [6-10]. The consumed energy and time are different for different walking paths. The longer walking path and the more turning times, the more energy and time consumed by the mowing robot. Considering the task of mowing robot and its blade center does not coincide with the motion center, it is not suitable to use random and spiral path planning algorithm. Roundabout path planning algorithm is simple, but the number of turns leads to lower efficiency. However, the mowing robot is mainly used in the large lawn of the airport. Compared with other open lawn environment, the large lawn of the airport has obvious characteristics, such as the static structure environment, no large obstacle and not allowed to be modified at will. Therefore, the paper proposes a new path planning algorithm focusing on the efficiency based on the traditional roundabout path planning algorithm.

The path planning adopts the method of polygon working area, and the autonomous walking mainly adopts the straight line without considering the curve way except the steering. The mowing robot collects the working area boundary location coordinates through the GPS, obtaining the polygon 
boundary vertices and boundary line equations. The angle of polygon working area is not more than $180^{\circ}$, otherwise it is split into multiple polygons. The first point of the polygon is set as the origin of coordinate, the connection of the first point and the second point is set as the $y^{\prime}$ axis shown in Fig. 6 . The coordinate transformation formula is as follows:

$$
\left[\begin{array}{l}
x_{i}^{\prime} \\
y_{i}^{\prime}
\end{array}\right]=\left[\begin{array}{cc}
\cos \gamma & -\sin \gamma \\
\sin \gamma & \cos \gamma
\end{array}\right]\left[\begin{array}{l}
x_{i}-x_{0} \\
y_{i}-y_{0}
\end{array}\right]
$$

Where $x_{i}$ and $y_{i}$ are respectively the coordinate value of the $i$-th point in the $o-x y$ terrestrial coordinate system, $x_{i}^{\prime}$ and $y_{i}^{\prime}$ are respectively the coordinate value of the $i$-th point in the $o^{\prime}-x^{\prime} y^{\prime}$ regional coordinate system, $x_{0}$ and $y_{0}$ are respectively the coordinate value of the first point in the $o$-xy terrestrial coordinate system, $\gamma$ is the angle between the $y$ axis and the $y^{\prime}$ axis, and clockwise is the positive direction.

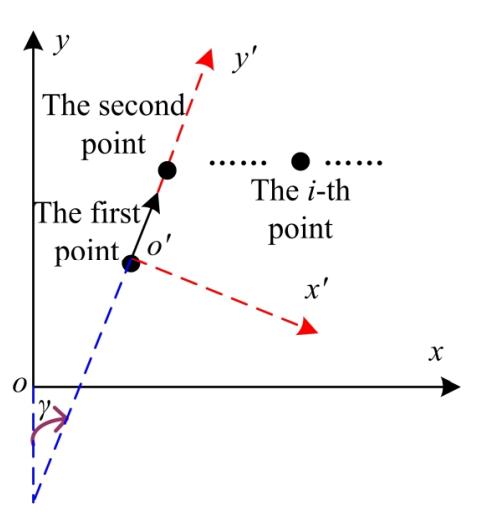

Fig. 6 Schematic diagram of coordinate transformation

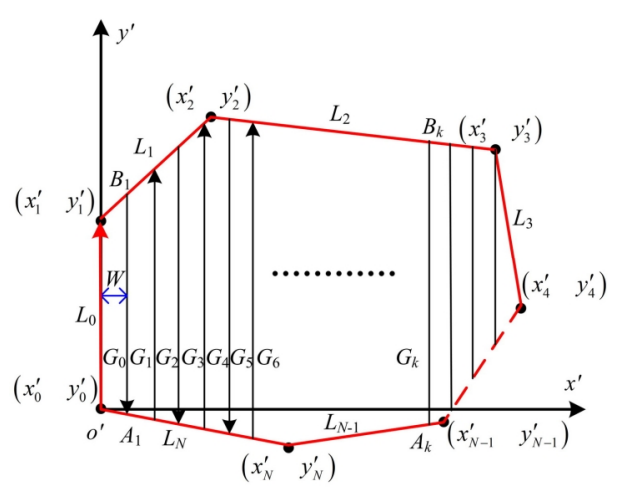

Fig. 7 Schematic diagram of polygon region planning

The path planning of roundabout type is simple and unified. The movement of mowing robot is easy to control, and the energy consumption is the minimum. It is the optimal path planning method for mowing robot. However, due to the intelligent mowing robot has a minimum turning radius, and the minimum turning radius is greater than half of the cutting table width, so if designing the path planning algorithm completely according to the traditional path planning algorithm will lead to miss cutting in the steering area. Therefore, the paper proposes the roundtrip linear path planning algorithm to complete the mowing work for robot.

Let the boundary point information of work area has been obtained by GPS, and completed the transformation calculation according to the equation (1). The coordinates of each point in the regional coordinate system are shown in Fig. 7. The planned path is the intersection of the straight line $G_{K}$ and the boundary line $L_{i}$. According to the coordinates of each point in the regional coordinate system, the boundary line $L_{i}$ in the regional coordinate system is divided into the following two cases.

Case 1. If $i \leq N-1$, the boundary line equation is as follows:

$$
\left(y_{i}^{\prime}-y_{i+1}^{\prime}\right) x^{\prime}+\left(x_{i+1}^{\prime}-x_{i}^{\prime}\right) y^{\prime}+\left(x_{i}^{\prime} y_{i+1}^{\prime}-x_{i+1}^{\prime} y_{i}^{\prime}\right)=0
$$

Case 2. If $i=N$, the boundary line equation is as follows:

$\left(y_{N}^{\prime}-y_{0}^{\prime}\right) x^{\prime}+\left(x_{0}^{\prime}-x_{N}^{\prime}\right) y^{\prime}+\left(x_{N}^{\prime} y_{0}^{\prime}-x_{0}^{\prime} y_{N}^{\prime}\right)=0$

The equation of the line $G_{K}$ in the regional coordinate system is $x^{\prime}=k W$, so the intersection of the straight line $G_{K}$ and the boundary line $L_{i}$ can be obtained by equations (2) and (3). After the coordinates of each intersection point are calculated, the path planning of mowing robot can be carried out according to the flow chart shown in Fig. 8.

Experimental analysis. Let boundary point information collected by GPS is as follows: $(0,0),(0,50)$, $(50,100),(120,50),(150,0),(50,-50)$. The boundary points are connected with straight line into a polygon working area. Through analysis found that the angles of polygon working area are all not more than $180^{\circ}$. According to the path planning algorithm, the results of path planning are shown in Fig. 9. 


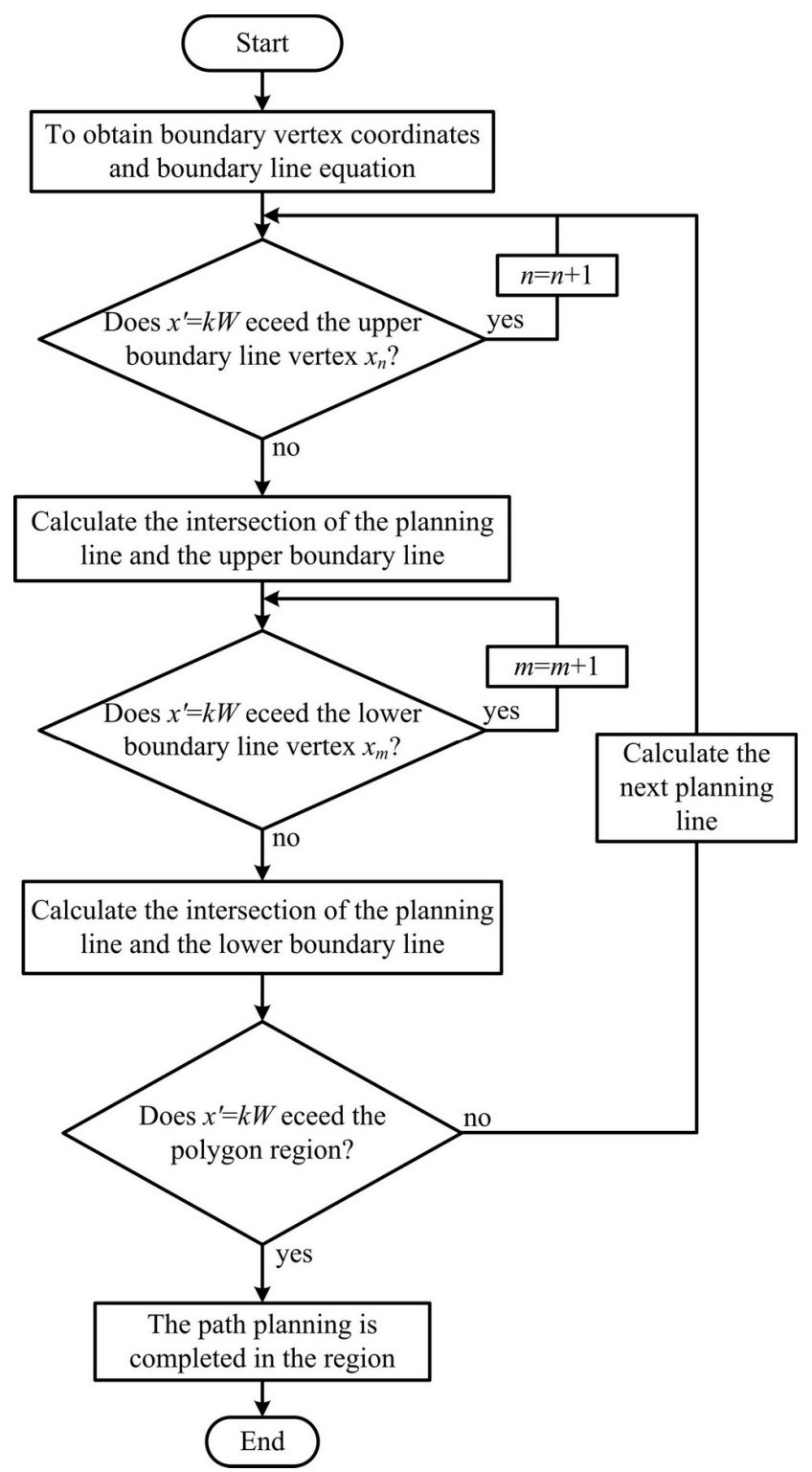

Fig. 8 Flow chart of line path planning in polygon region

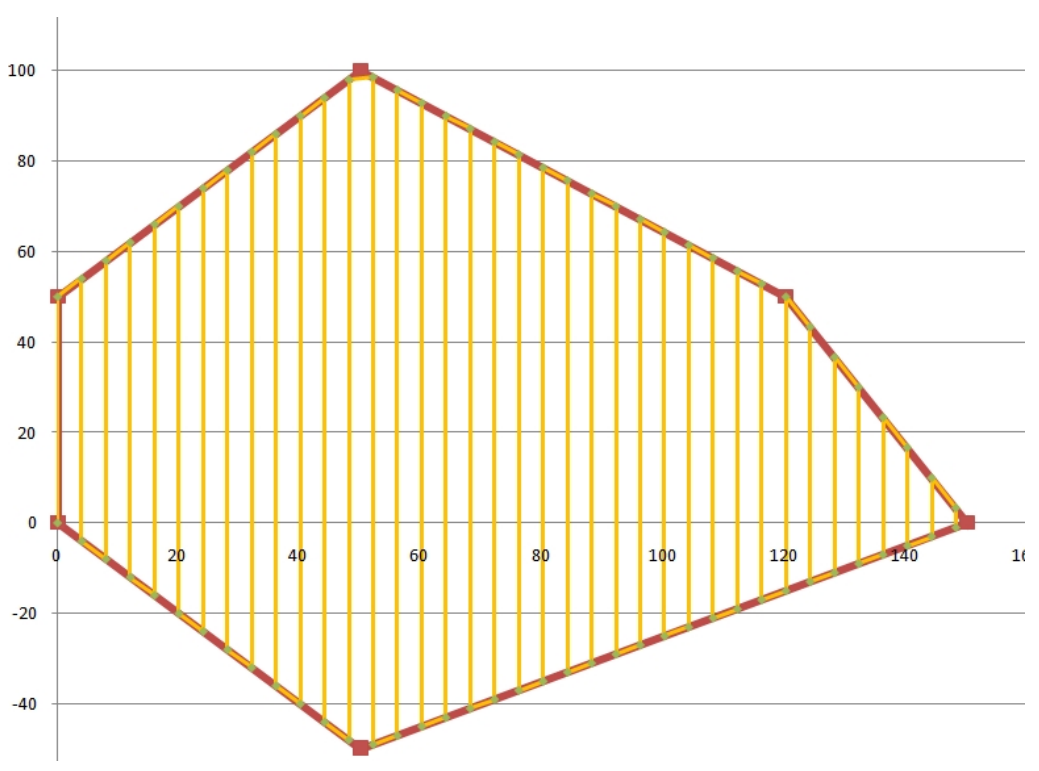

Fig. 9 Simulation experiment of path planning 


\section{Conclusions}

A new large mowing robot is designed for high efficiency mowing in large-scale lawn in the paper. The structure and functions of the mowing robot are introduced in detail from the system point of view. According to the position information collected by GPS on robot, the modeling of polygon working area is completed. The linear path equation of mowing robot is planned in the working area of polygon. Compared with the traditional roundabout path planning, it can overcome the defect of large leakage area in the steering area.

\section{Acknowledgements}

This work was financially supported by the National Basic Research Program of China (Grant No. 61663030), Postdoctoral Scientific Research Project of Jiangxi Province (Grant No. 2016KY35) and Project of Jiangxi Provincial Department of Education (Grant No.GJJ160694).

\section{References}

[1] WU Chao-feng and MA Xue-mei: Present situation and development of turf grass industry in the 21st century in China. Tianjin Agricultural Sciences, Vol. 15 (2009), pp. 74-77

[2] SHAN Hua-jia, LI Meng-lu, SUN Yan, et al: Recent development of turf grass industry in China. ACTA AGRESTIA SINICA, Vol. 21 (2013), pp. 222-229

[3] ZU Li: The research on area-covering control and dynamic characteristics for intelligent robot mower. PhD Thesis, Nanjing University of Science and Technology (2005)

[4] Zhang Xia and Cai Zongshou: Present situation and development countermeasures of lawn machinery in China. Journal of Agricultural Mechanization Research, Vol. 28 (2006), pp. 40-41

[5] CONG Ming, JIN Li-gang and FANG Bo: Intelligent robot mowers: a review. Robot, Vol. 29 (2007), pp. 407-416

[6] Zhu Daqi and Yan Mingzhong: Survey on technology of mobile robot path planning. Control and Decision, Vol. 25 (2010), pp. 961-967

[7] Xia Linlin, Zhang Jianpei and Chu Yan: An application survey on computational intelligence for path planning of mobile robots. CAAI Transaction on Intelligent Systems, Vol. 6 (2011), pp. $160-165$

[8] Li Renfu, Dokgo Myongchol, Hu Lin, et al: Mobile robot trajectory planning based on QPSO algorithm and experiment. Control and Decision, Vol. 29(2014), pp. 2151-2157

[9] Paolo Salaris, Daniele Fontanelli, Lucia Pallottino, et al: Shortest paths for a robot with nonholonomic and field-of-view constraints. IEEE Transactions on Robotics, Vol. 26 (2010), pp. 269-281

[10]XV Xing-jun: Design and simulation on regional all-covered algorithm of intelligent mower. Journal of Mechanical \& Electrical Engineering, Vol. 29 (2012), pp. 302-306 